\title{
A KINEMATIC MODEL OF THE HUMAN HAND TO EVALUATE ITS PREHENSILE CAPABILITIES
}

\author{
BRYAN BUCHHOLZ \\ Department of Work Environment, University of Massachusetts - Lowell, Lowell, MA, U.S.A. \\ and \\ THOMAS J. ARMSTRONG \\ Department of Environmental and Industrial Health, The University of Michigan, Ann Arbor, MI, \\ U.S.A.
}

\begin{abstract}
A kinematic model has been developed for simulation and prediction of the prehensile capabilities of the human hand. The kinematic skeleton of the hand is characterized by ideal joints and simple segments. Finger-joint angulation is characterized by yaw (abduction-adduction), pitch (flexion-extension) and roll (axial rotation) angles. The model is based on an algorithm that determines contact between two ellipsoids, which are used to approximate the geometry of the cutaneous surface of the hand segments. The model predicts the hand posture (joint angles) for power grasp of ellipsoidal objects by 'wrapping' the fingers around the object. Algorithms for two grip types are included: (1) a transverse volar grasp, which has the thumb abducted for added power; and (2) a diagonal volar grasp, which has the thumb adducted for an element of precision. Coefficients for estimating anthropometric parameters from hand length and breadth are incorporated in the model. Graphics procedures are included for visual display of the model. In an effort to validate the predictive capabilities of the model, joint angles were measured on six subjects grasping circular cylinders of various diameters and these measured joint angles were compared with angles predicted by the model. Sensitivity of the model to the various input parameters was also determined. On an average, the model predicted joint flexion angles that were $5.3 \%$ or $2.8^{\circ} \pm 12.2^{\circ}$ larger than the measured angles. Good agreement was found for the MCP and PIP joints, but results for DIP were more variable because of its dependence on the predictions for the proximal joints.
\end{abstract}

\section{INTRODUCTION}

Hand tools should be designed to minimize muscular effort and maximize grip strength capabilities in order to increase efficiency (Pheasant and O'Neill, 1975), reduce fatigue (Rohmert, 1973) and prevent mechanical trauma injuries (Tichauer and Gage, 1977; Silverstein et al., 1986). It is a well-known phenomenon that the strength of the hand is greatly influenced by the size of the object grasped (Hertzberg, 1955; Ayoub and LoPresti, 1971). This is usually attributed to the changing biomechanical advantage of the skeletal links as the joint angles change and also to the varying physiological advantage of the muscles as they change in length. The interaction of handle size and shape with hand anthropometry has a great effect on hand posture and, therefore, grip strength although in general the effects of this interaction on grip strength have not been considered. The objective of this research was the development of a predictive model for estimating the effects that anthropometry and object size have on prehensile hand posture, using circular cylinders in the first stage of this development.

Previous models of the hand, for the most part, have been developed for clinical applications and do not adapt well to tool design problems. Many of these models were constructed to predict the muscle and

Received in final form 21 June 1991. tendon forces used while grasping (Chao et al., 1976; Cooney and Chao, 1977; Berme et al., 1977; Toft and Berme, 1980), or to explore other important considerations in reconstructive surgery and orthotic design. These models are not well-suited for examining the effects of anthropometry and object geometry on the grip strength capabilities of the work population, although in theory these models may function in reverse, i.e. predict grip forces from muscle and tendon forces. None of the studies reviewed reported any capability for grip posture prediction.

Existing hand anthropometry and strength data are of great value to designers of industrial tasks and tools, but these data do not adapt well to specific situations (Armstrong, 1982). Few kinematic anthropometric data for the hand have been collected. An et al. (1979) have published data on the mean length of the proximal and middle phalangeal segments for the four fingers, but do not relate these data to the wrist joint nor do they locate the metacarpophalangeal (MCP) joints with respect to each other. Their data are important for comparing normal hand function to abnormal, but because of the interaction between the postures of the five digits the hand needs to be considered as a single kinematic-link structure for posture prediction. The model developed here deals with all of the segments of the hand together and employs statistically based hand anthropometry to predict hand posture. 
Empirical studies have looked at the effect of object size on grip strength using standard dynamometers (Hertzberg, 1955), electromyography (Ayoub and LoPresti, 1971), special dynamometers for measuring contributions from each of the phalanges (Amis, 1987) or psychophysical responses (Drury, 1980). Other authors state that hand-handle contact area should be maximized to reduce stress (Pheasant and O'Neill, 1975) and that there should be overlap between the thumb and index finger to provide the ability to resist grip breaking forces (Greenberg and Chaffin, 1977). The proposed model will make it possible to begin exploring the underlying mechanisms behind these empirical studies and to examine other more complex situations.

\section{DEVELOPMENT OF THE MODEL}

The model is kinematically based and uses ellipsoids to approximate the three-dimensional geometry of the cutaneous surface of the hand segments as well as object geometry. The model is built around an algorithm that determines contact between two ellipsoids developed by Fleck and Butler (1981) for the Calspan Crash Victim Simulator (CVS) and the U.S. Air Force Articulated Total Body (ATB) model. The model is capable of simulating the posture of the hand during any normal function. Procedures have been developed that are capable of predicting the hand posture for power grasp of an ellipsoidal object based on its size and basic orientation in the hand. The model is developed with the assumption that in a power grasp every segment of the hand will contact the object. Joint angles are determined by 'wrapping' the fingers around the object, beginning with the proximal segments and moving distally. The soft tissue deformation characteristics of the palmar hand are simulated using depth of penetration calculations included in this algorithm.

\section{Kinematic skeleton}

The kinematic skeleton of the human hand is mathematically approximated by ideal joints connected by simple line segments (Fig. 1). Joints and segments are numbered distally from the wrist joint. The nine interphalangeal joints (PIP, DIP and IP) are described as hinge joints capable of only flexion and extension. The five metacarpophalangeal joints (MCP) are saddle joints capable of both flexion-extension and abduction-adduction motions.

The carpometacarpal joint of the thumb (CMC) is described by many authors, e.g. Cooney and Chao (1977), as a saddle joint with two degrees of freedom. In reality, there is considerable rotation of the first metacarpal because of incongruity between the trapezium and the metacarpal base and laxity of the ligaments in the area (Haines, 1944; Kuczynski, 1974), although axial rotation is constrained to the flexion-extension and abduction-adduction motions and, therefore, is not considered a true degree of freedom (Cooney et al., 1981). For simplicity, the model describes the thumb CMC as having three degrees of freedom for movement.

For each segment, a local coordinate system is defined (Fig. 1) such that the proximal joint is the origin and the $x$-axis is defined as the vector from the proximal to the distal joint. The $y$-axis is projected dorsally perpendicular to the anterior-posterior plane for each segment, with the $z$-axis defined by the righthand rule. Therefore, the $z$-axis projects ulnarly for the right hand. The coordinates of the distal joint in the local coordinate system of the proximal joint are therefore $\left(r_{i j}, 0,0\right)$, where $r_{i j}$ is the length of the $j$ th segment of the $i$ th digit. For the root coordinate system, the $X$-axis is directed distally from the wrist joint center parallel to the third metacarpal. Therefore, the adjacent joints distal to the wrist joint have coordinates $\left(X_{i 1}, Y_{i 1}, Z_{i 1}\right)$, all of which may be nonzero.

The functionally equivalent wrist joint center described by Dempster (1955) is used in the model: 'On the palmar side of the hand, the distal wrist crease at the palmaris longus tendon, or the midpoint of a line between the radial styloid and the center of the pisiform bone; on the dorsal side of the hand, the palpable groove between the lunate and capitate bones, on a line with metacarpal bone III.' Other authors, e.g. Brunnstrom (1980) and Youm and Flatt (1980), located separate axes for flexion-extension and radio-ulnar deviation, with the axis for radio-ulnar deviation located slightly more distal. These centers are used as a convenient approximation, even though the rotations about the wrist are actually elliptical and cannot be idealized as simple levers with fixed centers (Taylor and Schwarz, 1955).

Cooney et al. (1981) found from ten cadaver hands that on an average the reference axes of the trapezium are flexed $48^{\circ}$, abducted $38^{\circ}$ and pronated (rotated medially) $80^{\circ}$ with respect to the reference axes of the third metacarpal. This relationship defines the neutral (zero) angular position of the thumb carpometacarpal joint. The model assumes that the neutral angular position of all other joints is aligned with the local coordinate system of the next proximal joint.

Movements (angular positions) are transferred proximally down the link structure to the root coordinate system at the wrist by homogeneous matrix multiplication. Chao et al. (1976) used what they called classical Eulerian angles to define finger-joint angulation. These angles can be described in terms of the physiological joint angles used by clinicians, as long as the order of rotations is considered. The abduction-adduction angle $(\theta$, yaw about the $y$-axis) is considered first, followed by flexion-extension ( $\phi$, pitch about the $z$-axis), and axial rotation ( $\psi$, roll about the $x$-axis) can be considered last. The matrix transforming a local coordinate system to the prox- 
imal system is therefore defined as

$\left[D_{i j}\right]=\left[\begin{array}{ccc}\mathrm{c} \phi_{i j} \mathrm{c} \theta_{i j} & -\mathrm{c} \psi_{i j} \mathrm{~s} \phi_{i j} \mathrm{c} \theta_{i j}+\mathrm{s} \psi_{i j} \mathrm{~s} \theta_{i j} & \mathrm{~s} \psi_{i j} \mathrm{~s} \phi_{i j} \mathrm{c} \theta_{i j}+\mathrm{c} \psi_{i j} \mathrm{~s} \theta_{i j} \\ \mathrm{~s} \phi_{i j} & \mathrm{c} \psi_{i j} \mathrm{c} \phi_{i j} & -\mathrm{s} \psi_{i j} \mathrm{c} \phi_{i j} \\ -\mathrm{c} \phi_{i j} \mathrm{~s} \theta_{i j} & \mathrm{c} \psi_{i j} \mathrm{~s} \phi_{i j} \mathrm{~s} \theta_{i j}+\mathrm{s} \psi_{i j} \mathrm{c} \theta_{i j} & -\mathrm{s} \psi_{i j} \mathrm{~s} \phi_{i j} \mathrm{~s} \theta_{i j}+\mathrm{c} \psi_{i j} \mathrm{c} \theta_{i j}\end{array}\right]$,

where $\mathrm{c}$ means cosine and $\mathrm{s}$ means sine.

\section{Segment contact bodies}

In order that hand-object contact points may be determined, the three-dimensional geometry of the hand segments are mathematically modelled as ellipsoids (Fig. 2). Ellipsoids are used to model the contact surfaces of ellipsoidal or spherical objects also, with elliptical cylinders defined as ellipsoids that have one very long $(\sim \infty)$ semi-axis that is truncated at the cylinder ends. The mathematical considerations of ellipsoid geometry are discussed in Appendix A.

Fleck and Butler (1981) have developed an efficient algorithm for determining contact, using ellipsoids as contact bodies, for the Calspan Crash Victim Simulator (CVS) and the U.S. Air Force Articulated Total Body (ATB) model. The mathematics used in this algorithm is given in Appendix B. This algorithm is based on the relationship between two ellipsoids that contact at a single point, i.e. the outward normal vectors from the two objects are parallel but in opposite directions. Fleck and Butler (1981) have also developed an ellipsoid-plane algorithm that could be used to determine contact between the hand segments and block-like objects.

\section{Power grasp algorithm and input requirements}

Napier (1956) divided prehension into two classes: precision grip and power grip. In precision grip, the object is pinched between the flexor aspects of one or more fingers and the opposing thumb. With power grip (Fig. 3), the object is held in a clamp formed by the fingers and the palm. The thumb may either be abducted, where it can reinforce the grasp, or adducted for an element of precision.

The model functions by reducing hand posture prediction to a series of one degree of freedom problems, i.e. all joint angles are initially set by the model and only the flexion-extension angle for a specified joint is allowed to vary at a given time. The model begins by flexing the proximal joints and proceeds distally, 'wrapping' the hand around the object. The model requires anthropometric data on segment lengths and the three-dimensional geometry of each segment contact body, although hand length and breadth are the only anthropometric data that are necessary as actual input.

Data from Buchholz (1989) are used in this analysis to estimate segment lengths and joint center locations based on linear models of hand length or breadth, but other population or individual data could be used. The kinematic skeleton of the hand that is used in the model is shown in Fig. 1. Segment lengths $\left(r_{i j}\right)$ are approximated as a proportion of hand length (HL):

$$
r_{i j}=C_{i j} \times \mathrm{HL} \pm \text { Error }
$$

The position of joint 2, i.e. thumb CMC and MCP of the four fingers, is described in $X-, Y$ - and $Z$-coordinates. The $X$-coordinate $\left(X_{i 1}\right)$ is modelled as a linear function of hand length $(\mathrm{HL})$ :

$$
X_{i 1}=F_{i 1} \times \mathrm{HL} \pm \text { Error, }
$$

and the $Z$-location $\left(Z_{i 1}\right)$ is modelled as a linear function of hand breadth (HB):

$$
Z_{i 1}=G_{i 1} \times \mathrm{HB} \pm \text { Error. }
$$

The coefficients $\left(C_{i j}, F_{i 1}\right.$ and $\left.G_{i 1}\right)$ for these models can be found in Buchholz (1989).

The $Z$-coordinate of MCP for digit III is defined as zero. For the right hand, the $Z$-coordinates of MCP for digits IV and $\mathrm{V}$ are positive and that for digit II negative. The $Y$-coordinates of the four finger MCP joints are estimated as zero, because adequate data are not available.

The necessary data for describing the hand segments as ellipsoids are shown in Fig. 2. The following linear models of hand breadth developed by Buchholz (1989) are used to approximate ellipsoid semi-axis breadth $\left(c_{i j}\right)$ and ellipsoid semi-axis depth $\left(b_{i j}\right)$, respectively:

$$
c_{i j}=H_{i j} \times \mathrm{HB} \pm \text { Error }
$$

and

$$
b_{i j}=K_{i j} \times \mathrm{HB} \pm \text { Error. }
$$

Also the coefficients $\left(H_{i j}\right.$ and $\left.K_{i j}\right)$ for these models can be found in Buchholz (1989).

The ellipsoid semi-axis length for the length of the segment $\left(a_{i j}\right)$ is arbitrarily estimated as $110 \%$ of the kinematic segment length:

$$
a_{i j}=0.55 r_{i j} \pm \text { Error. }
$$

It was estimated from a magnetic resonance image of a single index finger that about $60 \%$ of the soft tissue is located on the palmar side of the segments. This percentage corresponds to the following linear model for $l_{i j}$ :

$$
l_{i j}=0.2 b_{i j} \pm \text { Error. }
$$

The segment is assumed to be symmetric across its breadth.

Classification of the grip function of the hand is needed by the model to determine how to 'close' the 


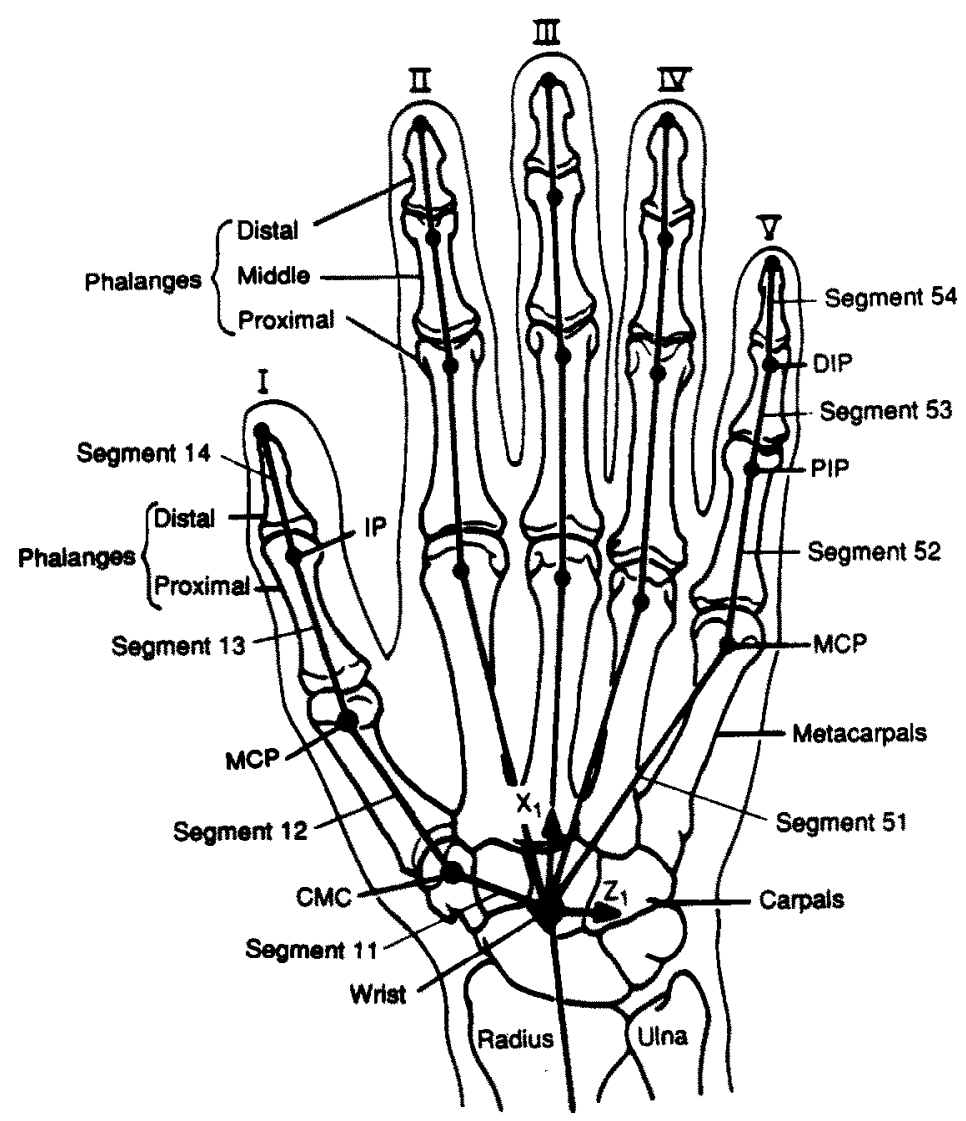

a)

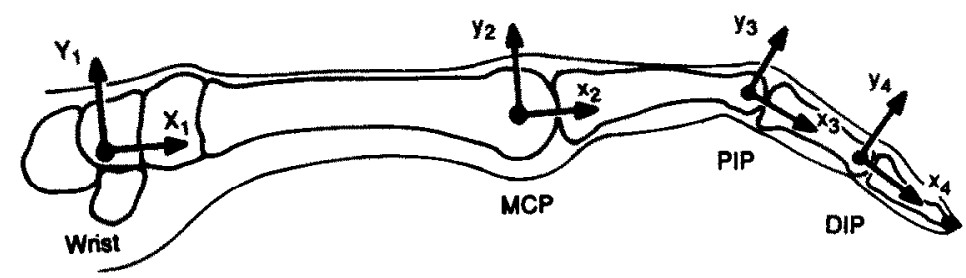

b)

Fig. 1. Kinematic skeleton of the human hand: (a) dorsal view; (b) ulnar view. The kinematic skeleton of the human hand is characterized by ideal joints separated by simple segments. Segments are numbered distally from the wrist joint. Local coordinate systems are identified for each joint with the origin located at the proximal joint center and the $x$-axis defined as the vector projecting toward the distal joint center. The $y$-axis projects dorsally so that flexion of the joint is a negative angle. The root coordinate system of the hand has an origin at the functional wrist joint center, with the $X$-axis pointing down the third metacarpal to the estimated position of the third MCP joint.

hand around the object. Sollerman (1980) divided power grasp into three categories: transverse volar grip, diagonal volar grip, and spherical volar grip. Figure 3 shows the two cylindrical grasps, the transverse and diagonal volar grips. The major differences in these two grasps are the object orientation and the thumb posture. For the transverse grasp the thumb is abducted and 'wrapped' around the object for increased power and with the diagonal grip the thumb is adducted and lies parallel to the object's long axis adding an element of precision.
The model is capable of predicting only flexionextension angles; therefore, all other joint angles need to be specified. Abduction-adduction for the finger MCP joints can usually be assumed to be zero, except for the spherical grasp where there is significant abduction of MCP, especially for the little finger. Abduction-adduction of both the CMC and MCP joints of the thumb need to be given as input. These will vary with cylinder size as well as grip function as described above. Pronation-supination (mediallateral rotation) of the thumb CMC also needs to be 


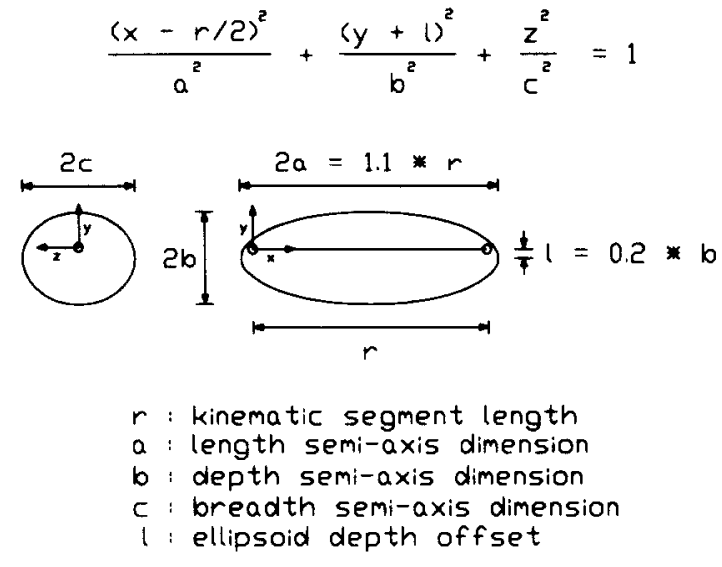

Fig. 2. Ellipsoidal description of the three-dimensional geometry of the hand segments. Ellipsoids are used to mathematically describe the three-dimensional geometry of the hand segments. Semi-axis lengths are needed to specify the size of the ellipsoid. The location of the center of the ellipsoid with respect to the origin of the kinematic segment is required, too.

specified. Increased pronation is noted when the thumb is abducted on large-diameter cylinders.

Soft tissue deformation is simulated in the model using the depth of penetration determined in the ellipsoid-ellipsoid contact algorithm. The depth of penetration criterion is introduced into the model as a strain, i.e. the ratio of the desired penetration depth to the depth of the segment is input. It is assumed that the strain on all segments is equal. Pilot work on one living hand indicates that the palmar tissue of the hand is initially very compliant and reaches a large deformation at low load and then stiffens so that a constant deformation may be assumed.

After the initial model data are set, the model estimates the location and orientation of the object in the root coordinate system. The $Z$-coordinate of the object center is specified as input and can often be set to zero. Cylindrical objects that have a long axis are oriented with their long axis at a given cylinder angle from parallel to the $Z$-axis of the hand (Fig. 3). The long axis is defined as the local $x$-axis of the object and $\theta$ is therefore defined by the following equation:

$$
\theta=90^{\circ} \text {-cylinder angle. }
$$

For cylinders with noncircular cross sections, a rotation about the long axis, $\psi$, may be specified.

The $X$-position of the object center may be specified or it can be estimated using the following empirically derived equations for power grasp of circular cylinders. The independent parameters in these equations are hand length (HL) and cylinder diameter (CD). The first equation predicts $X$-location for transverse volar grasps of circular cylinders:

$X_{\text {object }}=0.32 \times \mathrm{HL}-0.16 \times \mathrm{CD}+0.03 \times \mathrm{CD}^{2} \pm$ Error

and the second equation predicts $X$-location for diagonal volar grasps of circular cylinders:

$X_{\text {object }}=0.37 \times \mathrm{HL}-0.59 \times \mathrm{CD}+0.06 \times \mathrm{CD}^{2} \pm$ Error.

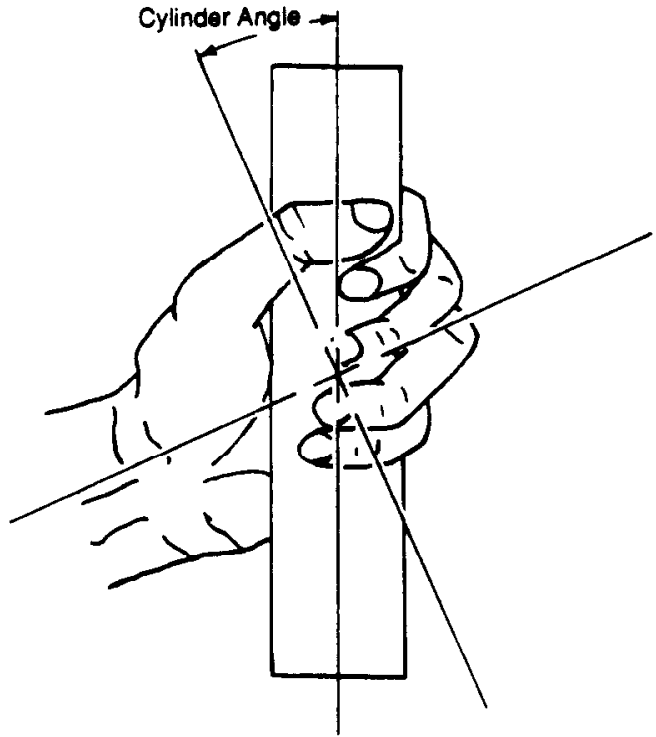

a)

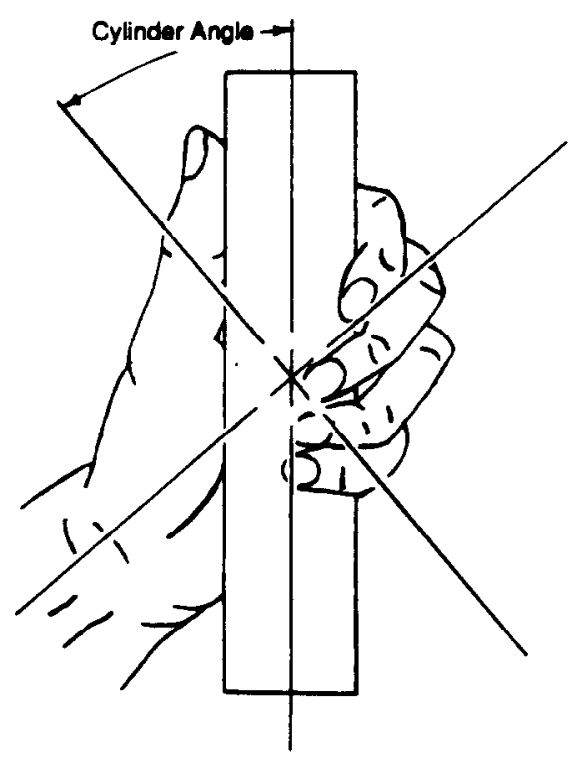

b)

Fig. 3. Power grasp of a circular cylinder. Algorithms for two different power grasps are included in the model. These are: (a) transverse volar grasp, with the thumb abducted for added power; (b) diagonal volar grasp, with the thumb adducted for an element of precision. Cylindrical objects are oriented with their long axis a given cylinder angle from parallel to the $Z$-axis of the hand.

Derivation of these equations is shown in Buchholz (1989).

The $Y$-location of the object center and $\phi$, rotation about the local $z$-axis, are then estimated by determining contact between the object and both the second and fifth metacarpal segments. The object is translated in the $Y$-direction and rotated in the negative $\phi$ - 
direction in small increments until the specified penetrations for the second and fifth metacarpals are reached.

After determining the location and orientation of the object, the next step is to determine the flexion-extension angles for the joints of the four fingers by 'wrapping' the finger around the object (Fig. 4). The model begins by flexing the MCP joint until the proximal phalange contacts the cylinder surface and the penetration constraint is met. Contact of the middle and distal phalanges must also be looked for, because there is the possibility that they will contact prior to the proximal segment. Next, the PIP joint is flexed until the middle (or distal) phalange comes into contact. The DIP is then flexed until the distal phalange deforms on the cylinder.

Two different algorithms have been developed for the two different thumb postures. The algorithm for the abducted thumb that contributes added power to the transverse grasp is similar to the finger algorithm. All of the CMC joint angles and the MCP abduction-adduction angles are given as initial input data. Then the MCP flexes until the proximal (or distal) phalange comes into contact with the object or any of the phalangeal segments of the index finger. The IP follows similarly, stopping when the distal phal-

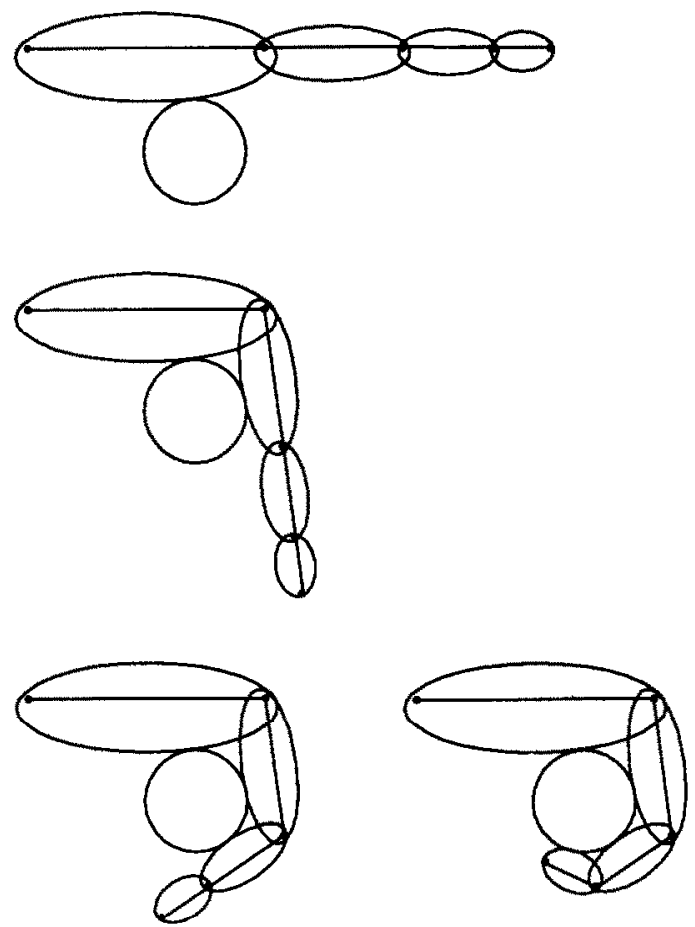

Fig. 4. Planar example of the power grasp algorithm. Initial posture is determined using the contact algorithm for the object and the metacarpal segments. The MCP abduction-adduction angle needs to be specified. The model then functions by 'wrapping' the hand around the object beginning with the proximal joints and proceeding distally. Each joint is flexed until the segment distal to it contacts the object and the penetration criteria are met. ange comes into contact with the object or the index finger.

The algorithm for the diagonal grip with the adducted thumb is somewhat simpler because it assumes that the MCP and IP joints are fully extended. The CMC joint is assumed to be in $10^{\circ}$ of pronation (medial rotation), while the abduction-adduction angles of the CMC and MCP joints are specified so that the thumb segments are aligned with the long axis of the cylinder (Fig. 3). The CMC joint of the thumb is then flexed until the distal phalangeal segment contacts the object.

Besides the flexion-extension joint angles of the hand, the model predicts the locations of the contact points and the orientations of the contact vectors and the ellipsoid penetrations that simulate the soft tissue deformation. Graphical display of the model is accomplished using routines developed for the ATB model (Leetch and Bowman, 1983). Ellipsoids are drawn as elliptical cross sections at equal intervals along the local $x$-axis of each of the segments and the object. These elliptical cross sections are drawn as a series of short vectors. Hidden line algorithms are included to provide a clear image. Figure 5 shows examples of this output for the transverse and diagonal volar grips.

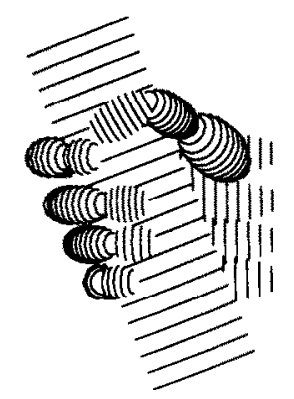

a)
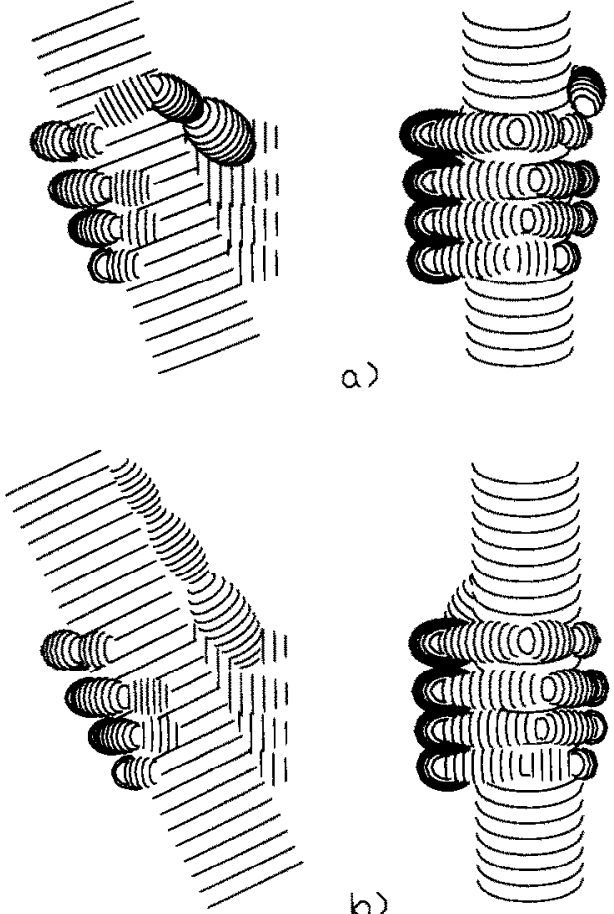

Fig. 5. Examples of the graphical display capabilities of the model. Different views of the (a) transverse volar grasp and (b) diagonal volar grasp are shown. Ellipsoids are drawn as a series of short vectors. Hidden line algorithms are included to provide a clear image, although these routines are costly in terms of processing time. 


\section{EVALUATION OF THE MODEL}

The ability of the model to predict the joint angles of the hand during prehension of circular cylinders has been evaluated as part of this research. Circular cylinders were chosen because they are readily available in various diameters and many tool handles have circular cross sections. Model validation was broken into three steps. These were:

(1) Collection of angular measurements in situ.

(2) Determination of the model's sensitivity to the various input parameters.

(3) Evaluation of the model's ability to predict flexion-extension joint angles.

\section{Collection of angular measurements in situ}

Flexion-extension joint angles were measured for three male and three female subjects holding various diameter circular cylinders in two power grasps: a transverse volar grasp [Fig. 3(a)] and a diagonal volar grasp [Fig. 3(b)]. Hands were chosen so that the range of hand length in the population was covered. Hand lengths ranged from first percentile female to ninety-fifth percentile male, where percentiles were determined from U.S. Air Force data (Garrett, $1970 \mathrm{a}, \mathrm{b})$.

Six cylinders were used, ranging in diameter from 1.60 to $7.61 \mathrm{~cm}$. The cylinders were cut to $\sim 16 \mathrm{~cm}$ in length from standard-sized aluminium tubing. Subjects were instructed to hold the cylinder comfortably in their hands and to not use excessive force. Grasps were demonstrated but subjects were allowed to determine the grasp specifics.

A manual finger goniometer (Preston, Corp., Clifton, NJ) was used to measure the joint angles. This goniometer consisted of two flat aluminium plates hinged together. One plate had a protractor attached to it and the other had a pointer which allowed angles to be read in $5^{\circ}$ increments. The plates were placed on the dorsal surface of the hand segments with the hinge centered over the joint.

Regression analysis was used to model the effects of hand length (HL) and cylinder diameter (CD) on the measured joint angles (JA). Separate regressions were used for each combination of digit, joint and grasp. Equations of the following form were tested:

$$
J A_{i j}=\mathrm{M} A_{i j}+\mathrm{M} B_{i j} \times \mathrm{HL}+\mathrm{M} C_{i j} \times \mathrm{CD} \pm \text { Error. }
$$

The results for both grasp types are similar. Measured flexion-extension angles for the joints of the four fingers are well modelled using the above linear equation. Coefficients of determination range between 0.41 and 0.94 , with larger $R^{2}$ for the transverse grasp. Cylinder diameter is the most important factor, with an increase in diameter causing decreased flexion of the joint. Significant $(p<0.001)$ coefficients for the cylinder diameter variable range from 3.19 to $10.0^{\circ} \mathrm{cm}^{-1}$ with cylinder diameter explaining between 22 and $94 \%$ of the variance in measured joint angles.
In all but four cases, increasing hand length increased joint flexion for the four fingers. The four exceptions were the MCP joints of digits II and III for both grips, which showed coefficients for decreased flexion with increasing hand length. Hand length accounts for between 0 and $69 \%$ of the variance in measured flexion angles.

The measured thumb joint flexion-extension angles are not described well using linear models of hand length and cylinder diameter. For the transver volar grasp, $R^{2}$ for the MCP is 0.43 and for the IP is 0.08 . Increasing hand length and cylinder diameter both have the effect of decreasing MCP joint flexion and increasing IP joint flexion for this grasp, although all coefficients for the IP joint are not significant $(p>0.1)$. For the diagonal volar grasp, a constant flexion of zero degree best describes these two thumb joints.

\section{Model sensitivity to input parameters}

The sensitivity of the model to the various input parameters was determined. The input parameters studied included the hand length, cylinder diameter, cylinder angle, ellipsoid penetration and the $X$-location of the object center. The hand lengths (and breadths) for the six subjects for whom joint angles were measured were used in the sensitivity analysis. The same cylinder diameters that were used in the angular measurement part of the validation study were employed here also.

Four cylinder angles were examined for each grasp type. For the transverse volar grasp, cylinder angles of $25,20,15$ and $10^{\circ}$ were used. For the diagonal volar grasp, the cylinder angles used were: $30,25,20$ and $15^{\circ}$. Four levels of ellipsoid penetration were examined. Strains of $0,10,20$ and $30 \%$ were investigated. Three $X$-locations for the object center were looked at: the empirically determined $X$-location and $X$-positions that were $\pm 0.5 \mathrm{~cm}$ from this empirically determined location.

All possible combinations of the above parameters were studied for the three joints of the four fingers using a batch setup of the model. The thumb was excluded because of its mobility, i.e. the need to set the abduction-adduction and axial rotation joint angles and the effect this would have on the sensitivity analysis.

Regression analysis was used to examine the sensitivity of the joint angles (JA) predicted by the model to variations in hand length (HL), cylinder diameter (CD), cylinder angle (CA), ellipsoid penetration (EP) and axial location $(X)$. Separate regressions were used for each combination of digit, joint and grasp. Equations of the following form were tested:

$$
\begin{aligned}
\mathrm{JA}_{i j}= & \mathrm{S} A_{i j}+\mathrm{S} B_{i j} \times \mathrm{HL}+\mathrm{SS} C_{i j} \times \mathrm{CD} \\
& +\mathrm{S} D_{i j} \times \mathrm{CA}+\mathrm{S} E_{i j} \times \mathrm{EP}+\mathrm{S} F_{i j} \times X \pm \text { Error }
\end{aligned}
$$

These linear regressions explained most of the variability in joint angle for MCP and PIP but not for DIP. For MCP, $R^{2}$ ranged from 0.86 to 0.93 , between 
0.78 and 0.83 for PIP and from 0.09 to 0.34 for DIP. The sensitivity of the model to the various input parameters was similar for both grip types.

Increasing the hand length resulted in a prediction of greater joint fiexion (Fig. 6). This effect was largest for the PIP joint in both grasps and for the MCP with the transverse grip. Sensitivity to this parameter was relatively small compared to other variables. For MCP with the transverse volar grasp, joint flexion was increased from 1.21 to $1.64^{\circ} \mathrm{cm}^{-1}$ increase in hand length. The values for PIP were between 1.85 and $3.45^{\circ} \mathrm{cm}^{-1}$. The sensitivities of the measured joint angles and those predicted by the model to hand length changes were similar.

Increasing cylinder diameter resulted in a prediction of decreased joint flexion (Fig. 7). Sensitivity to this parameter is relatively large. For MCP, joint flexion was decreased between 4.31 and $8.28^{\circ} \mathrm{cm}^{-1}$ increase in cylinder diameter. For PIP, these values ranged from 6.99 to $13.4^{\circ} \mathrm{cm}^{-1}$. The joint angles predicted by the model had a sensitivity to cylinder

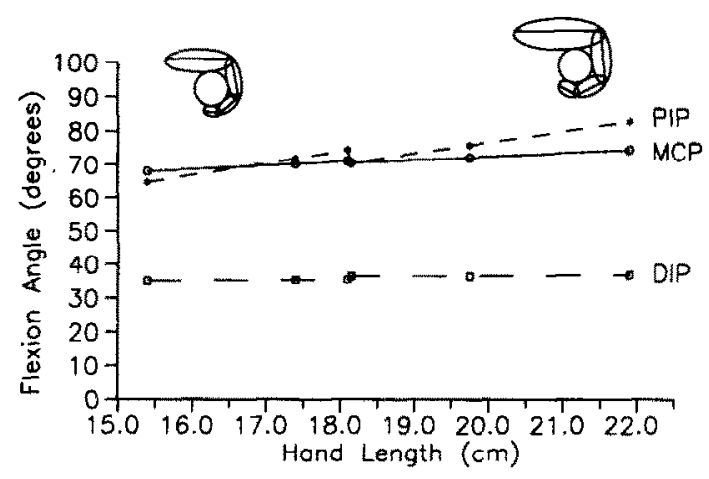

Fig. 6. The effect of hand length on joint angle prediction. Predicted angles averaged for four digits, six cylinder diameters, four cylinder angles, four ellipsoid penetrations, three axial locations and both grips ( $N=2304$ for each point) are shown for each joint as a function of hand length.

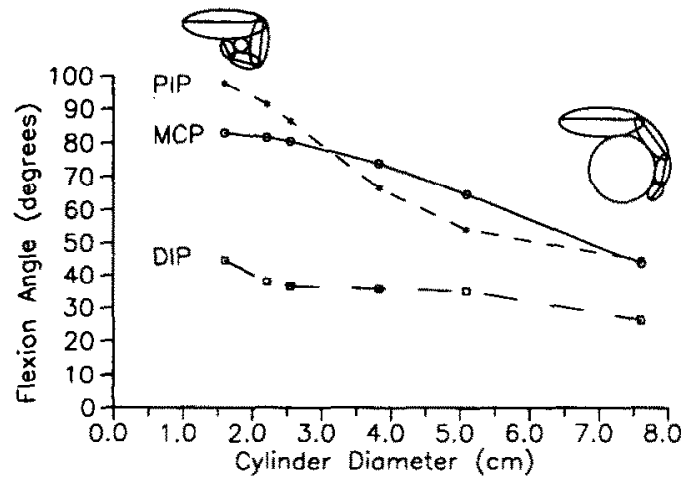

Fig. 7. The effect of cylinder diameter on joint angle prediction. Predicted joint angles averaged for four digits, six hand lengths, four cylinder angles, four ellipsoid penetrations, three axial locations and both grips ( $N=2304$ for each point) are shown for each joint as a function of cylinder diameter. diameter that was similar to that seen in the measured joint angles.

For MCP, increasing the cylinder angle (Fig. 3) decreased the amount of flexion for digits II and III and increased the flexion in digits IV and $V$, with the most pronounced effects at the second and fifth fingers (Fig. 8). The opposite effect was seen at PIP. For DIP, a slight decrease in flexion was seen with increasing cylinder angle at all four fingers.

Sensitivity of the predicted joint angles to ellipsoid penetration was relatively small for the range of penetrations examined (Fig. 9). Flexion increased between 0.43 and $0.73^{\circ}$ for a $1 \%$ increase in strain for PIP. For DIP, flexion decreases ranged from 0.03 to $0.55^{\circ}$ for a $1 \%$ increase in strain. For MCP, small increases in flexion were seen at digits III and IV and small decreases at $\mathrm{II}$ and $\mathrm{V}$.

The model is very sensitive to the $X$-location of the object center (Fig. 10). For MCP, joint flexion decreases ranged between 12.7 and $21.1^{\circ}$ per half $\mathrm{cm}$ distal move in object location. The opposite was seen at PIP. Flexion increased from 9.88 to $15.1^{\circ}$ per half $\mathrm{cm}$ distal move in object location. At DIP, smaller decreases in flexion were seen. These decreases ranged between 2.60 and $9.24^{\circ}$ per half $\mathrm{cm}$.

It is important to note that the sensitivity analysis is 'softened' by the fact that joint angles were limited to their normal range of motion. This is best seen in the sensitivity to ellipsoid penetration. Figure 9 shows that the model is not very sensitive to ellipsoid penetration, but the inset on Fig. 9 shows increased flexion at all joints for a strain of $40 \%$. This 'softening' occurs whenever the model inputs cause the hand posture to approach the flexion limits, i.e. when hand length is increased, cylinder diameter decreased, ellipsoid penetration increased and the $X$-location of the object is moved towards the wrist.

An important by-product of the joint angle prediction algorithm is the effect proximal joints angles have on the angles predicted for the more distal joints. A perturbation on the proximal joint moving it in one direction has the effect of moving the next distal joint in the opposite direction. For example, if for some reason MCP is caused to increase its flexion then PIP will decrease its flexion and DIP will increase its flexion. This 'zigzag' effect is seen in the sensitivity analysis of the model to the various input parameters and is probably best exemplified by the fact that predicted DIP joint angles have the most unexplained variance.

\section{Evaluation of the model's ability to predict joint angles}

The joint angles that were measured in situ were compared to joint angles predicted by the model for the same hand sizes and cylinder diameters. The empirically modelled $X$-locations of the object centers were used here. Cylinder angles were measured from photographs of the palmar views of the appropriate hand, cylinder and grasp. A strain level of $10 \%$ was used for all segments. 


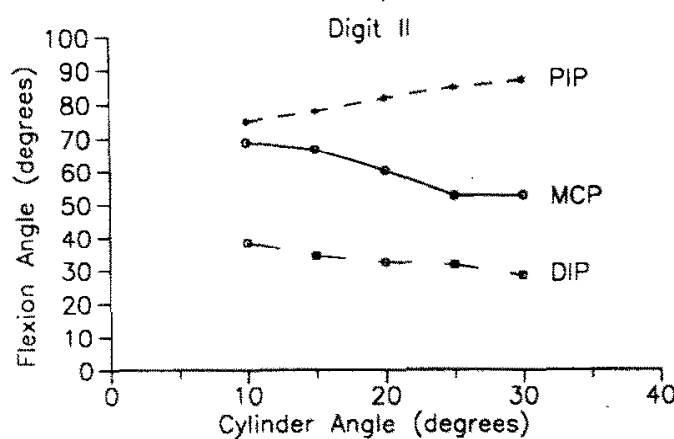

o)

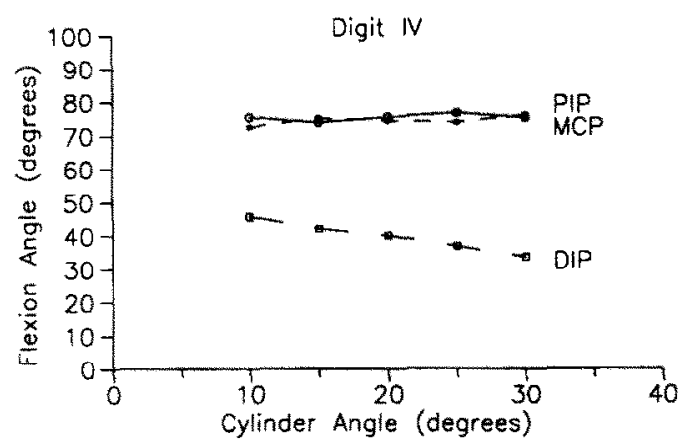

c)

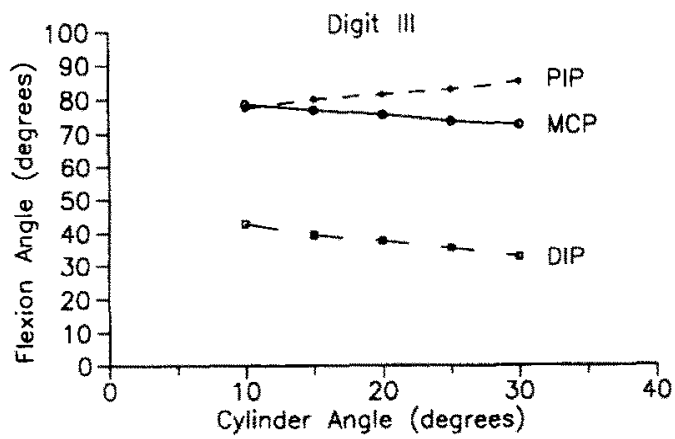

b)

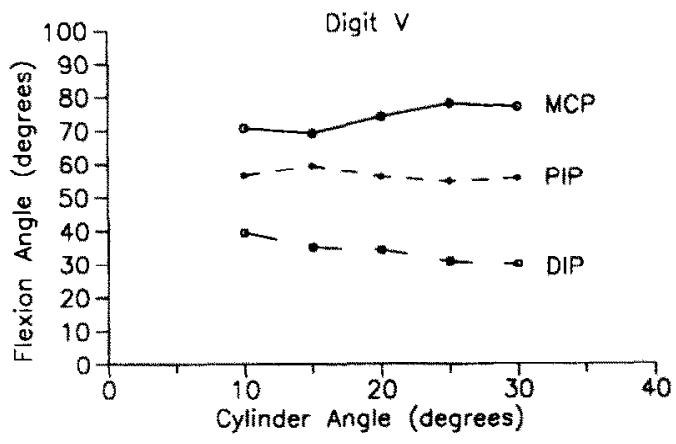

d)

Fig. 8. The effect of cylinder angle on joint angle prediction for (a) digit II, (b) digit III, (c) digit IV and (d) digit V. Predicted joint angles averaged for six hand lengths, six cylinder diameters, four ellipsoid penetrations, three axial locations and both grips ( $N=864$ for each point) are shown for each joint as a function of cylinder angle.

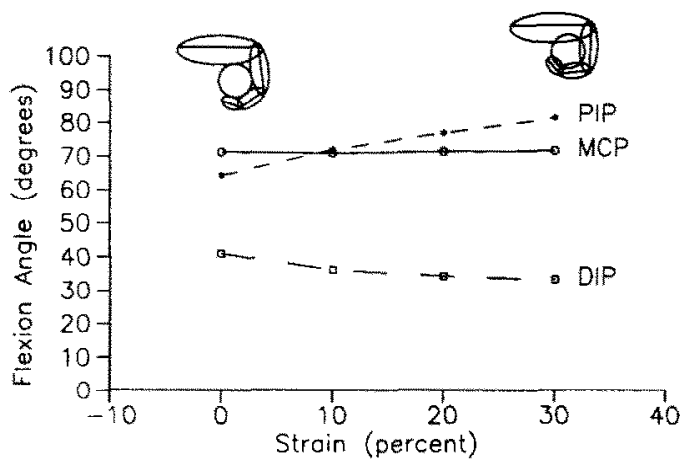

Fig. 9. The effect of ellipsoid penetration on joint angle prediction. Predicted joint angles averaged for four digits, six hand lengths, six cylinder diameters, four cylinder angles, three axial locations and both grips $(N=3456$ for each point are shown for each joint as a function of ellipsoid penetration.

For most of the joints, the model predicted more joint flexion than was measured (Table 1). This was true for all joints except the DIP joints of digits II and III with both grip types. The mean difference between the predicted and the measured joint angles varied between -11.3 and $+7.7^{\circ}$ for the transverse volar grasp, with the negative sign indicating that the pre-

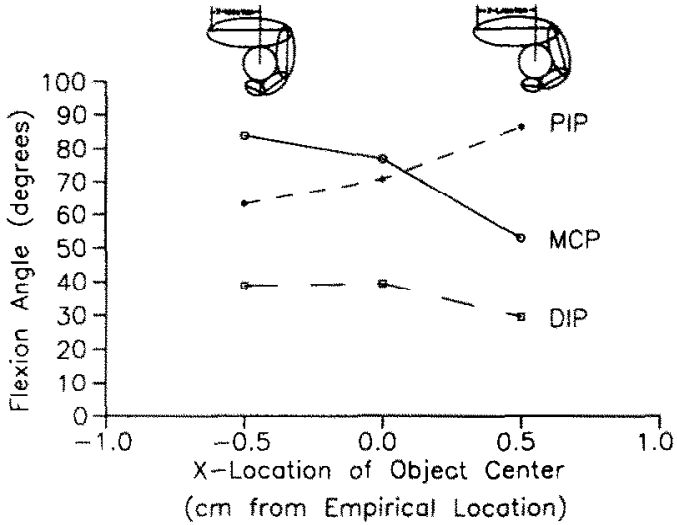

Fig. 10. The effect of axial location of the object center on joint angle prediction. Predicted joint angles averaged for four digits, six hand lengths, six cylinder diameters. Four cylinder angles, four ellipsoid penetrations and both grips ( $N=4608$ for each point) are shown for each joint, as a function of the axial location of the object center.

dicted flexion was larger than the measured flexion. For the diagonal grip, the mean difference ranged from -8.9 to $+5.7^{\circ}$

For the MCP and PIP, these predictions were 
Table 1. Pairwise comparison of predicted vs measured joint angles for both grasp types based on 6 hands and 6 cylinders $(N=36)$

\begin{tabular}{|c|c|c|c|c|c|c|c|}
\hline \multirow[b]{2}{*}{ Digit } & \multirow[b]{2}{*}{ Joint } & \multicolumn{3}{|c|}{ Transverse volar grasp } & \multicolumn{3}{|c|}{ Diagonal volar grasp } \\
\hline & & Predicted & Measured & Difference & Predicted & Measured & Difference \\
\hline I & $\begin{array}{l}\text { MCP } \\
\text { IP }\end{array}$ & $\begin{array}{l}-30.1 \\
-42.0\end{array}$ & $\begin{array}{l}-29.9 \\
-36.2\end{array}$ & $\begin{array}{l}-0.2 \pm 3.9(-0.7 \%) \\
-5.8 \pm 9.4(-16.0 \%)\end{array}$ & $\begin{array}{l}0.0 \\
0.0\end{array}$ & $\begin{array}{r}0.0 \\
-0.6\end{array}$ & $\begin{array}{l}0.0 \pm 0.0(-) \\
0.6 \pm 1.9(-)\end{array}$ \\
\hline II & $\begin{array}{l}\text { MCP } \\
\text { PIP } \\
\text { DIP }\end{array}$ & $\begin{array}{l}-69.5 \\
-78.2 \\
-28.4\end{array}$ & $\begin{array}{l}-62.8 \\
-71.7 \\
-36.0\end{array}$ & $\begin{array}{rr}-6.7 \pm 7.1 & (-10.7 \%) \\
-6.5 \pm 7.8 & (-9.1 \%) \\
7.7 \pm 11.2 & (21.4 \%)\end{array}$ & $\begin{array}{l}-62.6 \\
-77.2 \\
-35.4\end{array}$ & $\begin{array}{l}-59.9 \\
-68.8 \\
-41.0\end{array}$ & $\begin{array}{r}-2.7 \pm 8.2(-4.5 \%) \\
-8.5 \pm 10.4(-12.4 \%) \\
5.7 \pm 18.3 \quad(13.9 \%)\end{array}$ \\
\hline III & $\begin{array}{l}\text { MCP } \\
\text { PIP } \\
\text { DIP }\end{array}$ & $\begin{array}{l}-79.3 \\
-79.5 \\
-39.8\end{array}$ & $\begin{array}{l}-74.2 \\
-68.2 \\
-43.1\end{array}$ & $\begin{array}{rr}-5.1 \pm 5.2 & (-6.9 \%) \\
-11.3 \pm 4.3 & (-16.6 \%) \\
3.4 \pm 9.8 & (7.9 \%)\end{array}$ & $\begin{array}{l}-81.6 \\
-76.0 \\
-43.3\end{array}$ & $\begin{array}{l}-77.4 \\
-67.1 \\
-48.1\end{array}$ & $\begin{array}{rr}-4.3 \pm 9.9 & (-5.6 \%) \\
-8.9 \pm 6.8(-13.3 \%) & (-1.5 \pm 14.5 \\
4.9 & (10.2 \%)\end{array}$ \\
\hline IV & $\begin{array}{l}\text { MCP } \\
\text { PIP } \\
\text { DIP }\end{array}$ & $\begin{array}{l}-77.9 \\
-73.2 \\
-46.1\end{array}$ & $\begin{array}{l}-71.7 \\
-64.8 \\
-42.7\end{array}$ & $\begin{array}{l}-6.3 \pm 5.7(-8.8 \%) \\
-8.4 \pm 4.9(-13.0 \%) \\
-3.3 \pm 10.1 \quad(-7.7 \%)\end{array}$ & $\begin{array}{l}-82.8 \\
-66.9 \\
-48.9\end{array}$ & $\begin{array}{l}-78.4 \\
-62.4 \\
-45.4\end{array}$ & $\begin{array}{ll}-4.4+7.3 & (-5.6 \%) \\
-4.5 \pm 5.8 & (-7.2 \%) \\
-3.5 \pm 18.5 & (-7.7 \%)\end{array}$ \\
\hline V & $\begin{array}{l}\text { MCP } \\
\text { PIP } \\
\text { DIP }\end{array}$ & $\begin{array}{l}-75.3 \\
-53.6 \\
-43.6\end{array}$ & $\begin{array}{l}-71.5 \\
-52.1 \\
-39.1\end{array}$ & $\begin{array}{l}-3.9 \pm 6.1 \quad(-5.5 \%) \\
-1.5 \pm 7.4(-2.9 \%) \\
-4.5 \pm 19.8(-11.5 \%)\end{array}$ & $\begin{array}{l}-82.7 \\
-43.1 \\
-43.6\end{array}$ & $\begin{array}{l}-80.9 \\
-46.9 \\
-41.7\end{array}$ & $\begin{array}{ll}-1.8 \pm 7.4 & (-2.2 \%) \\
-3.9 \pm 13.9 & (-8.3 \%) \\
-1.9 \pm 31.7 & (-4.6 \%)\end{array}$ \\
\hline \multicolumn{2}{|c|}{$\begin{array}{l}\text { Average } \\
(N=504)\end{array}$} & -58.3 & -54.6 & $3.7 \pm 10.0 \quad(-6.8 \%)$ & -53.2 & -51.3 & $-1.8 \pm 14.0 \quad(-3.5 \%)$ \\
\hline
\end{tabular}

consistent for the hands and cylinders examined. Differences were largest at the extremes of cylinder size, i.e. for cylinders with diameters of 1.60 and $7.61 \mathrm{~cm}$. The difference between predicted and measured joint angles showed the most variance for the DIP joint, due to the 'zigzag' effect.

The worst predictions were for the DIP joint of digit $\mathrm{V}$ with the diagonal grasp, where the model predicts too little joint flexion for the smaller cylinders and for cylinders larger than $3.83 \mathrm{~cm}$ the model predicts more flexion than is measured. Predicted flexion is less than measured flexion for the PIP joint on the larger cylinders and this contributes to the effect at the DIP joint. A similar result is seen for DIP.V with the transverse grip, although differences between measured and predicted angles for DIP-V are not as large for this grip type, indicating that cylinder angle probably contributes to these differences.

\section{DISCUSSION}

The objective of this research was the development of a predictive model for estimating the effects that anthropometry and object size have on prehensile hand posture. Predicting hand posture is important for tool handle design because of its effect on grip strength capabilities. Previous authors have examined the effect of object size on grip strength using various empirical methods, but few have looked at the effect of hand anthropometry on grip strength. None of the studies reviewed reported any capability for predicting or quantifying grip posture. In this study, joint angles were both empirically measured and predicted using a kinematic model of the hand.

The model usually predicted more joint flexion than was measured for the same hand and cylinder. This could be due, in part, to limitations in the model's capabilities. Most of these are related to the lack of adequate data and the assumptions that were made in order to fill some of the input requirements. The model is also limited to the prediction of flexion-extension angles, and other joint angles must be estimated. The other limitation is that the model has been evaluated only with circular cylinders. In theory, the model is capable of predicting hand posture on ellipsoidal objects, but this has to be evaluated before arbitrary handle shapes can be examined. These limitations are discussed in the next section, followed by a description of future work with the model.

\section{Model limitations}

Buchholz (1989) collected a large amount of anthropometric data for use in this model, nevertheless there are some holes in these data and assumptions were made in an attempt to fill these holes. In general, the data for predicting the location of the joints of the kinematic skeleton from hand length and breadth are good for the $x$ - and $z$-dimensions (standard errors below $1 \mathrm{~mm}$ ), although an overestimation of these lengths would increase the amount of flexion predicted. However, data were not collected on the $Y$ locations of the finger MCP joints and these were estimated as zero. The $Y$-location of MCP-III is defined as zero but the other MCP are located at most $5 \mathrm{~mm}$ volar of MCP-III, with MCP-V the most volar. The effect due to this assumption should be relatively small.

Buchholz (1989) indicated that ellipsoids were not the most accurate description of the cutaneous surface of the segments. Buchholz collected data that would allow modelling the surface of each segment as a group of ellipsoids instead of a single ellipsoid, to more 
accurately depict segment geometry, although this would increase processing time for the model. The length of the ellipsoids was arbitrarily chosen to be $110 \%$ of the kinematic segment length to enhance graphical depiction. Ellipsoid length may have a small effect on the cross-sectional shape of the ellipsoid but the effect on posture prediction should be minimal. There is no reason, other than increased complexity, that different methods should not be used for depicting segment geometry depending upon the desired result or use, e.g. different methods could be used for posture prediction and graphical depiction.

The anthropometry for modelling the cutaneous surface of the segments as ellipsoids was collected using calipers, so it was not possible to relate these measurements to the actual kinematic segment. The assumption that $60 \%$ of the soft tissue is located palmar of the kinematic segment was made using the magnetic resonance image of the phalanges of a single index finger. It is not known if there is any variation between digits, for the carpometacarpal segments or between people. This could potentially have a large effect on posture prediction. Locating the kinematic segment volarly with respect to the center of the ellipsoid would increase the flexion that the model predicts and vice versa.

Assumptions were made about soft tissue deformation so that it could be simulated using ellipsoid penetration calculations made by the model. Pilot work on one living hand indicated that the palmar tissue of the hand is initially very compliant and reaches a large deformation at low load and then stiffens, so that a constant deformation may be assumed. Good quantitative data were not collected. A strain of $10 \%$ was used for all segments, although actual soft tissue deformation may be different for each segment. A strain of $10 \%$ may be too large and could have led to increased predictions of joint flexion. Actual soft tissue deformations need to be measured for all of the segments of the hand.

The model employs empirical equations for locating the object along the $X$-axis of the hand. These equations appear to be adequate for use with circular cylinders. Predicted flexion was greater than measured flexion at both MCP and PIP, so it is unlikely that changing the $X$-location of the object would improve the results in that MCP and PIP would be affected oppositely. This is a limitation though, because the model is very sensitive to this location and this method will probably not adapt well to other object shapes. Using contact between the object and the thumb metacarpal segment may provide an accurate method for locating the object, but more knowledge of the thumb kinematics is required before this can happen.

The model is limited to the prediction of flexion-extension angles, and other joint angles must be estimated. Zero MCP abduction-adduction was assumed for the grasp of circular cylinders. Observations suggest that this is probably an adequate estimate of normal hand posture for the transverse grasp. As the cylinder angle increases, it appears that the $\mathrm{MCP}$ joints ulnarly deviate. For other objects, e.g. a baseball, significant abduction of the MCP joints, especially for digit $\mathrm{V}$, may occur. The ability of these joints to abduct decreases with increasing flexion due to the action of ligaments, so that zero abduction is a good approximation for small objects. Empirical estimation of abduction-adduction may be necessary for some objects.

The mobility of the thumb creates even more difficulty. The thumb is capable of abduction-adduction at both MCP and CMC and axial rotation at CMC and these angles need to be estimated. A detailed empirical study of the kinematics of the thumb during power grasp is needed. Cooney et al. (1981) did some work in this area and their data were helpful in positioning the thumb, but their study lacked detail for power grasps.

The other limitation in the model is that it has been evaluated only with circular cylinders. Evaluation with various ellipsoidal objects needs to be accomplished before tool handles may be examined. This evaluation and removal of the other model limitations will obviously improve model accuracy and usefulness and are obviously important considerations for future work.

\section{Future research}

The kinematic model is capable of predicting hand postures on circular cylinders and, with evaluation, will also predict hand postures on various ellipsoidal objects. The model will then be used to examine hand tool designs from a best hand-tool fit point of view. A hand tool that is too small for a given hand will cause the joint angles to approach their flexion limits. An object that is too large will not provide sufficient overlap between the thumb and fingers for resisting breakaway forces (Greenberg and Chaffin, 1977).

It is also expected that this model will be used in biomechanical modelling of the hand to predict the forces involved in prehension. The model could be used as a framework for attaching tendons and muscles, so that grip forces may be evaluated as a function of hand anthropometry and object geometry. Buchholz et al. (1988) describe a pilot study using this kinematic model with a planar kinetic model of the muscle and tendon force capabilities of the index finger developed by Wells et al. (1985) for predicting grip strengths on circular cylinders. Favorable comparisons between predicted grip strengths and strengths measured by Amis (1987) were found.

The model was developed with ergonomic applications in mind, but could also be used to examine clinical orthopaedic conditions. For example, the effects of degenerative joint diseases or joint implants on grip and pinch postures could be analyzed. The model could be used to help design tools and utensils for people with a limited range of finger flexion. The shape of these handles could be modified so that the maximum contact between the hand and object occurs, 
increasing frictional forces and strengthening the grasp.

A predictive model for estimating the effects that anthropometry and object size have on prehensile hand posture has been developed, using circular cylinders initially. The model is kinematically based and uses ellipsoids to approximate the three-dimensional geometry of the cutaneous surface of the hand segments as well as object geometry. Future work on the model will allow posture prediction for complexshaped objects. The model will be used to examine the effects of hand anthropometry and object size and shape on hand posture and grip strength and will have important applications in tool design and grip strength prediction.

Acknowledgements - This research was supported by the U.S. National Aeronautics and Space Administration (NASA training grant number NGT-23-005-802 in conjunction with the NASA Center of Excellence in Man-Systems Research at the University of Michigan), the National Institute for Occupational Safety and Health and by gifts from AMP Corporation, Harrisburg, PA. The authors thank Ints Kaleps from the Biodynamics and Bioengineering Division of the Harry G. Armstrong Aerospace Medical Research Lab at Wright-Patterson Air Force Base for access to the Articulated Total Body model.

\section{REFERENCES}

Amis, A. A. (1987) Variation of finger forces in maximal isometric grasp tests on a range of cylinder diameters. $J$. Biomed. Engng 9, 313-320.

An, K. N., Chao, E. Y., Cooney, W. P. and Linscheid, R. L. (1979) Normative model of human hand for biomechanical analysis. J. Biomechanics $12,775-788$.

Armstrong, T. J. (1982) Development of a biomechanical hand model for study of manual activities. In Anthropometry and Biomechanics: Theory and Application (Edited by Easterby, R., Kroemer, K. H. E., and Chaffin, D. B.), pp. 183-191. Plenum, New York.

Ayoub, M. M. and LoPresti, P. (1971) The determination of an optimum size cylindrical handle by use of electromyography. Ergonomics 14, 509-518.

Berme, N., Paul, J. P. and Purves, W. K. (1977) A biomechanical analysis of the metacarpophalangeal joint J. Biomechanics 10, 409-412.

Brunnstrom, S. (1980) Clinical Kinesiology. Davis, Philadelphia, Ch. 4, pp. 85-133.

Buchholz, B. O. (1989) A kinematic model of the human hand to evaluate its prehensile capabilities. Ph.D. dissertation The University of Michigan, Ann Arbor, MI

Buchholz, B., Wells, R. P. and Armstrong, T. J. (1988) The influence of object size on grasp strength: results of a computer simulation of cylindrical grasp. Twelfth Annual Conference of the American Society of Biomechanics, Urbana-Champaign, IL.

Chao, E. Y., Opgrande, J. D. and Axmear, F. E. (1976) Threedimensional force analysis of finger joints in selected isometric hand functions. J. Biomechanics 9, 387-396.

Cooney, W. P. and Chao, E. Y. S. (1977) Biomechanical analysis of static forces in the thumb during hand function. J. Bone Jt Surg. 59A, 27-36.

Cooney, W. P., Lucca, M. J., Chao, E. Y. S. and Linscheid, R. L. (1981) The kinesiology of the thumb trapeziometacarpal joint. J. Bone Jt Surg. 63A, 1371-1381.

Dempster, W. T. (1955) Space requirements of the seated operator-geometrical, kinematic, and mechanical aspects of the body with special reference to the limbs. Report No. WADC-TR-55-159, Wright Air Development Center, Air research and Development Command, Wright-Patterson Air Force Base, Ohio.

Drury, C. G. (1980) Handles for manual materials handling. Appl. Ergonomics 11, 35-42.

Fleck, J. T. and Butler, F. E. (1981a) Validation of the Crash Victim Simulator, Vol. 1, Engineering Manual Part I: Analytical Formulation. National Technical Information Service, Springfield, VA.

Fleck, J. T. and Butler, F. E. (1981b) Validation of the Crash Victim Simulator, Vol. 2, Engineering Manual Part II: Validation Effort. National Technical Information Service, Springfield, VA.

Fleck, J. T. and Butler, F. E. (1981c) Validation of the Crash Victim Simulator, Vol. 3, User's Manual. National Technical Information Service, Springfield, VA.

Fleck, J. T. and Butler, F. E. (1981d) Validation of the Crash Victim Simulator, Vol. 4, Programmer's Manual. National Technical Information Service, Springfield, VA.

Garrett, J. W. (1970a) Anthropometry of the hand of female air force flight personnel. Report No. AMRL-TR-69-26, Aerospace Medical Research Laboratory, Aerospace Medical Division, Air Force Systems Command, Wright-Patterson Air Force Base, $\mathbf{O H}$.

Garrett, J. W. (1970b) Anthropometry of the hands of male air force flight personnel. Report No. AMRL-TR-69-42, Aerospace Medical Research Laboratory, Aerospace Medical Division, Air Force Systems Command, Wright-Patterson Air Force Base, $\mathrm{OH}$.

Greenberg, L. and Chaffin, D. B. (1977) Workers and Their Tools: A Guide to the Ergonomic Design of Hand Tools and Small Presses. Pendell, Midland, MI.

Haines, R. W. (1944) The mechanism of rotation at the first carpo-metacarpal joint. $J$. Anat. 78, 44-46.

Hertzberg, H. T. E. (1955) Some contributions of applied physical anthropology to human engineering. Ann. N.Y. Acad. Sci. 63, 616-629.

Kuczynski, K. (1974) Carpometacarpal joint of the human thumb. J. Anat. 118, 119-126.

Leetch, B. D. and Bowman, W. L. (1983a) Articulated Total Body (ATB) "View" Program Software Report, Part I, Programmer's Guide. Report No. AFAMRL-TR-81-111, Biodynamics and Bioengineering Division, Air Force Aerospace Medical Research Laboratory, Aerospace Medical Division, Air Force Systems Command, Wright-Patterson Air Forc Base, $\mathrm{OH}$

Leetch, B. D. and Bowman, W. L. (1983b) Articulated Total Body (ATB) “View" Program Software Report, Part II, User's Guide. Report No. AFAMRL-TR-81-111, Biodynamics and Bioengineering Division, Air Force Aerospace Medical Research Laboratory, Aerospace Medical Division, Air Force Systems Command, Wright-Patterson Air Force Base, $\mathrm{OH}$.

Napier, J. R. (1956) The prehensile movements of the human hand. J. Bone Jt Surg. 38B, 902-913.

Pheasant, S. and O'Neill, D. (1975) Performance in gripping and turning. Appl. Ergonomics 6, 205-208.

Rohmert, W. (1973) Problems in determining rest allowances, Part 1: Use of modern methods to evaluate stress and strain in static muscular work. Appl. Ergonomics 4, 91-95.

Silverstein, B. A., Fine, L. J. and Armstrong, T. J. (1986) Hand wrist cumulative trauma disorders in industry. Br. J. Ind. Med. 43, 779-784.

Sollerman, C. (1980) Grip function of the hand: analysis, evaluation and a new test method. Section of Hand Surgery, Department of Orthopaedic Surgery, Sahlgren Hospital, University of Göteborg, Göteberg, Sweden.

Taylor, C. L. and Schwarz, R. J. (1955) The anatomy and mechanics of the human hand. Artif. Limbs 2, 22-35.

Tichauer, E. R. and Gage, H. (1977) Ergonomic principles basic to hand tool design. Am. Ind. Hygiene Assoc. J. 38, $622-634$. 
Toft, R. and Berme, N. (1980) A biomechanical analysis of the joints of the thumb. J. Biomechanics 13, 353-360.

Wells, R. P., Ranney, D. A. and Keeler, A. (1985) The interaction of muscular and passive elastic forces during unloaded finger movements: a computer graphics model. In Biomechanics: Current Interdisciplinary Research (Edited by Perren, S. M. and Schneider, E.), pp. 743-748. Martinus Nijhof, Dordrecht.

Youm, Y. and Flatt, A. E. (1980) Kinematics of the wrist. Clin. Orthop. Rel. Res. 117, 24-32.

\section{APPENDIX A \\ MATHEMATICAL CONSIDERATIONS OF ELLIPSOID GEOMETRY}

Fleck and Butler (1981) discuss the mathematical considerations of ellipsoid geometry. Consider an ellipsoid (Fig. 2) whose principal axes are aligned with the local coordinate system. The points on the cllipsoid satisfy the relation

$$
[[r]-[D]]^{T}[A][[r]-[I]]=1 \text {, }
$$

where $T$ denotes the transpose and signifies the dot product, and

$$
\begin{aligned}
& {[r]=\left[\begin{array}{l}
x \\
y \\
z
\end{array}\right], \quad[]=\left[\begin{array}{c}
r / 2 \\
-l \\
0
\end{array}\right],} \\
& {[A]=\left[\begin{array}{ccc}
1 / a^{2} & 0 & 0 \\
0 & 1 / b^{2} & 0 \\
0 & 0 & 1 / c^{2}
\end{array}\right] .}
\end{aligned}
$$

For convenience, let the center of the ellipsoid be at the origin of the local coordinate system, i.e. $[I]=[0]$. Then, the ellipsoid equation is written as

$$
[r]^{\mathrm{T}}[A][r]=1 .
$$

If the local coordinate system $[r]$ is rotated by the direction cosine matrix $[D]$ such that

$$
[r]=[D][s],
$$

then

$$
\begin{aligned}
{[r]^{\mathrm{T}}[A][r] } & =[s]^{\mathrm{T}}[D]^{\mathrm{T}}[A][D][s] \\
& =[s]^{\mathrm{T}}\left[[D]^{\mathrm{T}}[A][D]\right][s] \\
& =[s]^{\mathrm{T}}[B][s]=1,
\end{aligned}
$$

where

$$
[B]=[D]^{\mathrm{T}}[A][D]
$$

is the matrix describing an ellipsoid whose principal axes are oriented by the rotation specified by $[D]$ with respect to the local system of $[s]$.

\section{APPENDIX B \\ ELLIPSOID-ELLIPSOID CONTACT ALGORITHM}

Fleck and Butler (1981) have developed an algorithm for efficiently determining the contact between two ellipsoids. Because this reference is not readily available, the mathematics for this algorithm is given here.

Consider the case of two ellipsoids, $A$ and $B$, that just touch in a single point (Fig. 11). The basic geometrical relations are:

$$
\begin{aligned}
v \mu\left[A_{\mathbf{A}}\right][x] & =-[n], \\
\mu\left[A_{\mathbf{B}}\right]([x]-[m]) & =[n],
\end{aligned}
$$

where $\left[A_{\mathrm{A}}\right]$ is the matrix describing ellipsoid $\mathrm{A},\left[A_{\mathrm{B}}\right]$ is the matrix describing ellipsoid $B,[x]$ is the vector from the center of ellipsoid $A$ to the point of contact, $[m]$ is the vector from

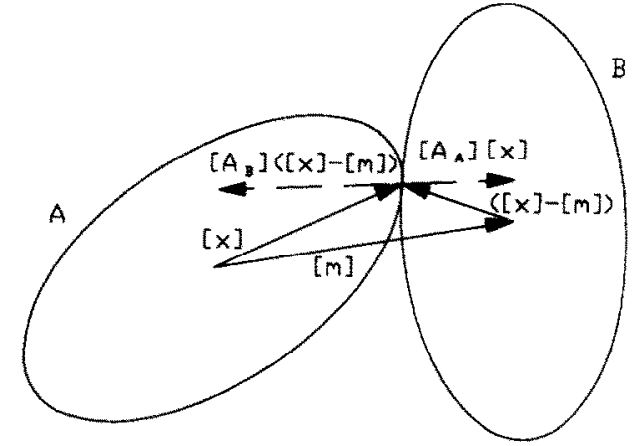

Fig. 11. Ellipsoid-ellipsoid contact algorithm (from Fleck and Butler, 1981). The algorithm is based on the relationship between two ellipsoids that contact in a single point i.e. the outward normal vectors from the two ellipsoids are parallel but in opposite directions.

the center of ellipsoid A to the center of ellipsoid $\mathrm{B},[n]$ is the outward normal from ellipsoid $B$, and $v$ and $\mu$ are scalars.

Therefore,

$$
v\left[A_{A}\right][x]=-\left[A_{\mathbf{B}}\right]([x]-[m]),
$$

which yields

$$
\left(v\left[A_{\mathrm{A}}\right]+\left[A_{\mathrm{B}}\right]\right)[\mathrm{x}]=\left[A_{\mathrm{B}}\right][\mathrm{m}] .
$$

Thus, the single point of contact $[x]$ is determined by the parameter $v$.

The basic equations of the ellipsoids are:

$$
[x]^{\mathrm{T}}\left[A_{\mathrm{A}}\right][x]=1,
$$$$
\left[[x]-[m]^{\mathrm{T}}\left[A_{\mathrm{B}}\right][x]-[m]\right]=1 \text {. }
$$

Let

$$
\begin{gathered}
f_{\mathrm{A}}(v)=[x]^{\mathrm{T}}\left[A_{\mathrm{A}}\right][x], \\
f_{\mathrm{B}}(v)=[[x]-[m]]^{\mathrm{T}}\left[A_{\mathrm{B}}\right][[x]-[m]] .
\end{gathered}
$$

For a particular value of $[x]$, if $f_{\mathrm{A}}(v)>1$, then $[x]$ is outside the ellipsoid; if $f_{A}(v)<1$, then $[x]$ is inside the ellipsoid. Now define the function $g(v)$ such that

$$
g(v)=f_{A}(v)-f_{B}(v) \text {. }
$$

The single point of contact is then determined as the point where $g(v)=0$.

Investigation of the equations shows that solving for $v$ where $g(v)=0$ is equivalent to solving a sixth-degree polynomial in $v$. Rather than solving the polynomial, a Newton-Raphson procedure is used, where $g(v)$ is expanded in a Taylor series as follows:

$$
g(v+\mathrm{d} v)=g(v)+\delta v \mathrm{~d} g /\left.\mathrm{d} v\right|_{v}
$$

Since it is desired that $g(v+\mathrm{d} v)=0$,

$$
\delta v=-g(v) /\left(\mathrm{d} g /\left.\mathrm{d} v\right|_{v}\right)
$$

This procedure is iterated until a specific degree of convergence is achieved $(|\delta v / v|<\varepsilon)$ or until a specified number of steps have been executed and convergence has failed, in which case an error message is printed.

The initial value of $v$ is estimated as

$$
v=\left([m]^{\mathrm{T}}\left[A_{\mathrm{B}}\right][m] /[m]^{\mathrm{T}}\left[A_{\mathrm{A}}\right][m]\right)^{1 / 2}
$$

This produces a $v$ of about the right order of magnitude.

Using the expressions for $f_{\mathrm{A}}$ and $f_{\mathrm{B}}$, the following equations result:

$$
\begin{gathered}
\mathrm{d} f_{\mathrm{A}} / \mathrm{d} v=2(\mathrm{~d}[x] / \mathrm{d} v) \cdot\left[A_{\mathrm{A}}\right][x], \\
\mathrm{df}_{\mathrm{B}} / \mathrm{d} v=-v \mathrm{~d} f_{\mathrm{A}} / \mathrm{d} v, \\
\mathrm{~d}[x] / \mathrm{d} v=-\left[v\left[A_{\mathrm{A}}\right]+\left[A_{\mathrm{B}}\right]\right]^{-1}\left[A_{\mathrm{A}}\right][x], \\
\mathrm{d} g / \mathrm{d} v=\mathrm{d} f_{\mathrm{A}} / \mathrm{d} v-\mathrm{d} f_{\mathrm{B}} / \mathrm{d} v=(1+v) \mathrm{d} f_{\mathrm{A}} / \mathrm{d} v .
\end{gathered}
$$


When the solution is obtained an expansion factor is defined as

$$
e_{\mathrm{f}}=\left(f_{\mathrm{A}}\right)^{1 / 2}
$$

The expansion factor is used to determine the depth of penetration. The single point of contact $[x]$ is defined for an ellipsoid that has been contracted. The location of the same point on $A$ when not contracted is

$$
\left[x_{\mathrm{A}}\right]=[x] / e_{\mathrm{f}},
$$

and on $B$ is

$$
\left[x_{\mathrm{B}}\right]=[[x]-[m]] / e_{\mathrm{f}}+[m]
$$

The vector between these points is

$$
\left[x_{\mathrm{A}}\right]-\left[x_{\mathrm{B}}\right]=\left(1 / e_{\mathrm{f}}-1\right)[m] .
$$

The depth of penetration is taken as the magnitude of this vector.

$$
p=\left(1 / e_{\mathrm{f}}-1\right)|[m]| .
$$

The depth of penetration is used to simulate soft tissue deformation during deformation hand posture prediction. 